\title{
SEPARATING CONJUGATES IN AMALGAMATED FREE PRODUCTS AND HNN EXTENSIONS
}

\author{
JOAN L. DYER \\ (Received 14 June 1978; revised 14 May 1979) \\ Communicated by $\mathbf{H}$. Lausch
}

\begin{abstract}
A group $G$ is termed conjugacy separable (c.s.) if any pair of distinct conjugacy classes may be mapped to distinct conjugacy classes in some finite epimorph of $G$. The free product of $A$ and $B$ with cyclic amalgamated subgroup $H$ is shown to be c.s. if $A$ and $B$ are both free, or are both finitely generated nilpotent groups. Further, one-relator groups with nontrivial center and HNN extensions with c.s. base group and finite associated subgroups are also c.s.
\end{abstract}

1980 Mathematics subject classification (Amer. Math. Soc.): primary 20 E 26, 20 E 06; secondary 20 F 10.

\section{Introduction}

A group is conjugacy separable (c.s.) if, whenever $x$ and $y$ are not conjugate in $G$, there is a finite quotient group of $G$ in which the images of $x$ and $y$ are not conjugate. In this paper we shall prove that the free product of the groups $A$ and $B$ with amalgamated subgroup $H$ is c.s. if $A$ and $B$ are c.s. and $H$ is finite, or if $A$ and $B$ are finitely generated nilpotent groups and $H$ is cyclic, or if $A$ and $B$ are free groups and $H$ is cyclic. These results extend work if Lipschutz (1966) and of Stebe (1968, $1970,1971)$. It is my pleasure to acknowledge that conversations with them, M. Anshel and G. Baumslag led to my interest in these problems.

We shall also establish that one-relator groups with nontrivial center, and HNN extensions of c.s. groups with finite associated subgroups, are c.s. The first of these results is due to S. M. Armstrong (1977); that both follow from Dyer (1979) was communicated by D. J. Collins (in letters), and the proofs follow his suggestions. 
The main theorem of Dyer (1979) is that free-by-finite groups are c.s. Since an amalgamated free product $(A * B: H)$ with finite factors $A$ and $B$ is free-by-finite (Neumann (1954)), as is an HNN extension $\left(G, t: t^{-1} H t=K\right)$ with finite base group $G$ (Karrass et al. (1973)), we have

THEOREM 1. If $A, B$ and $G$ are finite groups, then $(A * B: H)$ and $\left(G, t: t^{-1} H t=K\right)$ are both c.s.

Observe that, whenever any pair of nonconjugate elements in the group $G$ can be mapped to nonconjugate elements in a c.s. image of $G$, then $G$ is itself c.s. Moreover, using theorems of Solitar and Collins (stated below as Theorems 2 and 3 ), we can determine when two elements either of an amalgamated free product or of an HNN extension are conjugate. This puts us in a position to exploit Theorem 1. The pattern of argument is similar to that used by G. Baumslag (1963) to establish the residual finiteness of certain amalgamated free products of nilpotent groups.

In Section 2, after establishing notation, we recall some facts about amalgamated free products and HNN extensions and state the conjugacy theorems; full statements of the results asserted above and their proofs occupy Section 3.

The final version of this paper was prepared while the author was a Visiting Scientist at the Thomas J. Watson Research Center, I.B.M., under the auspices of the Mathematics Department.

\section{Preliminaries}

For $x, y$ elements of $G$ and $S$ a subset of $G$, write $x \sim_{S} y$ if there exists an $s \in S$ such that $x=s^{-1} y s$ and $x \sim_{S} y$ otherwise. Let $\{x\}^{G}$ be the conjugacy class $\left\{y: y \sim_{G} x\right\}$. Let $\left\{S_{i}\right\}$ be a family of pairwise disjoint subsets of $G$ and $\alpha: G \rightarrow L$ be an epimorphism. Say that $\alpha$ separates the $S_{i}$ if the $\alpha\left(S_{i}\right)$ are pairwise disjoint subsets of $L$. In particular, $x \in G$ is conjugacy distinguished (c.d.) in $G$ if $x x_{G} y$ implies that there is an epimorphism $\alpha: G \rightarrow L$ that separates $\{x\}^{G}$ from $\{y\}^{G}$ where $L$ is a finite group. Hence $G$ is c.s. if and only if all its elements are c.d.

Let $P$ be the free product of $A$ and $B$ with amalgamated subgroup $H$; we write

$$
\begin{aligned}
P & =(A * B: H), \quad \text { or } \\
& =(A * B: A \leftarrow H \rightarrow B)
\end{aligned}
$$

where $H \rightarrow A$ and $H \rightarrow B$ are injective homomorphisms. We shall view $A$ and $B$ as subgroups of $P$ with $H=A \cap B ; P$ satisfies a universal mapping property with 
respect to pairs of homomorphisms $A \rightarrow G, B \rightarrow G$ which agree on $H$. Each element of $P$ may be written as an alternating product

$$
u_{1} u_{2} \ldots u_{k}
$$

where $u_{i} \in A \cup B$ and if $k \geqslant 2$, successive syllables belong to different factors of $P$ (that is, either $u_{i} \in A-H$ and $u_{i+1} \in B-H$, or else $u_{i} \in B-H$ and $u_{i+1} \in A-H$, for each $i=1,2, \ldots, k-1)$. Call the alternating product $u_{1} u_{2} \ldots u_{k}$ cyclically reduced if $k=1$ or if $k \neq 1$ and $u_{k}, u_{1}$ also belong to different factors of $P$. Each product

$$
u_{i} \ldots u_{k} u_{1} \ldots u_{i-1}
$$

is a cyclic permutation of $u_{1} u_{2} \ldots u_{k}$ to which it is conjugate in $P$, and is alternating if $u_{1} u_{2} \ldots u_{k}$ is cyclically reduced. Note that $\{x\}^{P}$ always contains cyclically reduced elements. The length of an alternating product is defined as follows:

$$
u_{1} u_{2} \ldots u_{k}= \begin{cases}0 & \text { if } k=1 \text { and } u_{1} \in H \\ 1 & \text { if } k=1 \text { and } u_{1} \in(A \cup B)-H \\ k & \text { otherwise. }\end{cases}
$$

If $u_{1} \ldots u_{k}$ and $v_{1} \ldots v_{j}$ are alternating products and

$$
u_{1} \ldots u_{k}=v_{1} \ldots v_{j}
$$

then $j=k$ and there exists a sequence

$$
h_{1}, h_{2}, \ldots, h_{k-1}
$$

of elements in $H$ such that

$$
u_{1}=v_{1} h_{1}^{-1}, \quad u_{2}=h_{1} v_{2} h_{2}^{-1}, \quad \ldots, \quad u_{k-1}=h_{k-2} v_{k-1} h_{k-1}^{-1}, \quad u_{k}=h_{k-1} v_{k}
$$

Observe that $u_{1} \ldots u_{k}$ is cyclically reduced if and only if $v_{1} \ldots v_{k}$ is, and that length is defined on $P$.

The criterion for conjugacy in an amalgamated free product is the following:

THEOREM 2 (Magnus et al. (1966), p. 212). Let $P=(A * B: H)$ and let $x \in P$ be of minimal length in its conjugacy class. Suppose $y \in P, y$ is cyclically reduced, and $y \sim{ }_{P} x$.

(1) If $\|x\|=0$, then $\|y\| \leqslant 1$ and if $y \in A$ say, there is a sequence $h_{1}, h_{2}, \ldots, h_{r}$ of elements in $H$ such that

$$
y \sim{ }_{A} h_{1} \sim{ }_{B} h_{2} \sim{ }_{A} \cdots \sim_{B} h_{r}=x .
$$


(2) If $\|x\|=1$, then $\|y\|=1$ and either $x \in A, y \in A$ and $x \sim{ }_{A} y$, or else $x \in B$, $y \in B$ and $x \sim_{B} y$.

(3) If $\|x\| \geqslant 2$, then $\|y\|=\|x\|$ and $y \sim_{B} x^{*}$ where $x^{*}$ is a cyclic permutation of $x$.

Note that any cyclically reduced element of $P$ is of minimal length in its conjugacy class except for elements of length one that are conjugate to elements in $H$ :

$$
a \in A-H \text { and }\{a\}^{A} \cap H \neq \varnothing \text {, }
$$

or

$$
b \in B-H \text { and }\{b\}^{B} \cap H \neq \varnothing .
$$

Finally, observe that if $x=u_{1} \ldots u_{k}$ and $y=v_{1} \ldots v_{k}$ where both products are alternating, then $x \sim_{H} y$ if and only if there exists a finite sequence $h_{0}, h_{1}, \ldots, h_{k}$ of elements in $H$ such that

$$
\left\{\begin{array}{c}
u_{1}=h_{0}^{-1} v_{1} h_{1}, \\
u_{2}=h_{1}^{-1} v_{2} h_{2}, \\
\vdots \\
u_{k}=h_{k-1}^{-1} v_{k} h_{k}
\end{array}\right.
$$

and

$$
h_{k}=h_{0} \text {, }
$$

for (I) and (II) are equivalent to $x=h_{0}^{-1} y h_{0}$ with $h_{0} \in H$ (see Stebe (1971), Lemma 8).

The structure of HNN extensions parallels that of amalgamated free products and will be employed in a similar fashion below (see Miller (1971)). Let $Q$ be the HNN extension $\left(G, t: t^{-1} H t=K\right)$. Here $G$ is the base group, $H$ and $K$ the associated subgroups, and $H \rightarrow K$ is a fixed isomorphism effected by conjugation by $t$ within $Q$. Each element of $Q$ may be written as a $t$-reduced product

$$
g_{0} t^{e(1)} g_{1} \ldots t^{e(k)} g_{k} \text {, }
$$

where $g_{i} \in G, e(i)= \pm 1$, and no subwords $t^{-1} h t(h \in H)$ or $t k t^{-1}(k \in K)$ occur. Such a product has length $k$; length is well defined on $Q$, and will be denoted as above by a double bar. Herein, we term a product cyclically reduced if it is of length zero or else is a $t$-reduced product of the form

$$
t^{e(1)} g_{1} \ldots t^{e(k)} g_{k}
$$

all of whose cyclic permutations

$$
t^{e(i)} g_{i} \ldots t^{e(k)} g_{k} t^{e(1)} g_{1} \ldots t^{e(i-1)} g_{i-1}
$$

are also $t$-reduced. The notation $u \sim \sim_{G, t *} v$ will be used for elements $u, v \in G$ to mean either $u \sim_{Q} v$, or $u \in H$ and $v=t^{-1} u t(\in K)$, or $u \in K$ and $v=t u t^{-1}(\in H)$. 
THEOREM 3 (Collins (1969) or see Miller (1971) p. 21). Let $x$ and $y$ be cyclically reduced elements of $Q$, and suppose that $x \sim_{Q} y$. Then $\|x\|=\|y\|$, and one of the following holds:

(1) $\|x\|=\|y\|=0$ and there is a finite sequence of elements $z_{1}, z_{2}, \ldots, z_{m}$ in $H \cup K$ such that

$$
x \sim_{G} z_{1} \sim_{G, l^{*}} z_{2} \sim{ }_{G, l^{*}} z_{3} \ldots \sim_{G, l^{*}} z_{m} \sim_{G} y
$$

(2) $\|x\|=\|y\| \geqslant 1$ and $y \sim_{H \cup K} x^{*}$ where $x^{*}$ is a cyclic permutation of $x$.

Note that any element of $Q$ is conjugate to some cyclically reduced element which is of minimal length in its conjugacy class. Finally, if $x=t^{e(1)} u_{1} \ldots t^{e(k)} u_{k}$ and $y=t^{f(1)} v_{1} \ldots t^{f(k)} v_{k}$ are both cyclically reduced products, then $x \sim_{H} \cup{ }_{K} y$ if and only if

$$
e(i)=f(i) \text { for } i=1, \ldots, k
$$

and there is a finite sequence of elements $z_{0}, \ldots, z_{k}$ in $H \cup K$ for which

$$
t^{-e(i)} z_{i-1} t^{e(i)}=w_{i} \in H \cup K \text { for } i=1, \ldots, k,
$$

$$
\left\{\begin{array}{c}
u_{1}=w_{1}^{-1} v_{1} z_{1} \\
u_{2}=w_{2}^{-1} v_{2} z_{2} \\
\vdots \\
u_{k}=w_{k}^{-1} v_{k} z_{k}
\end{array}\right.
$$

and

$$
z_{k}=z_{0}
$$

for these equations are equivalent to $x=z_{0}^{-1} y z_{0}$ with $z_{0} \in H \cup K$. (The associated subgroup to which $z_{i}$ belongs is determined by the sign of $e(i)$.)

\section{The proofs}

Our first result concerns the free product $\boldsymbol{P}$ of c.s. groups with a finite amalgamated subgroup. Stebe (1971) proved that cyclically reduced elements of length at least two in $\boldsymbol{P}$ are c.d., by reducing first to the case in which the factors are finite in a fashion dictated by Theorem 2 , and then by producing the requisite finite image. The presence of Theorem 1 shortens this argument, and a complete proof is included.

THEOREM 4. If $A$ and $B$ are c.s. and $H$ is finite, then $(A * B: H)$ is c.s. 
Proof. Since $A$ and $B$ are residually finite, the families

$$
\mathscr{M}=\{M: M \triangleleft A,|A / M|<\infty, H \cap M=1\}
$$

and

$$
\mathscr{N}=\{N: N \triangleleft B,|B / N|<\infty, H \cap N=1\}
$$

satisfy the two conditions

$$
\bigcap_{M \in \mathscr{M}} M=1=\bigcap_{N \in \mathscr{N}} N
$$

and

$$
\bigcap_{M \in \mathscr{M}} M H=H=\bigcap_{N \in \mathscr{N}} N H .
$$

For any $(M, N) \in \mathscr{M} \times \mathscr{N}$, the natural projections $A \rightarrow A / M$ and $B \rightarrow B / N$ extend to an epimorphism $\pi_{M, N}: P \rightarrow P_{M, N}$ where $P=(A * B: H)$ and

$$
P_{M N}=(A / M * B / N: A / M \leftarrow H \rightarrow B / N)
$$

(with, for example, $H \cong H / H \cap M \simeq H M / M \rightarrow A / M$ naturally). The groups $P_{M, N}$ are c.s. by Theorem 1 .

Note that $\mathscr{K}$ and $\mathscr{N}$ are closed under intersections with normal subgroups of $A$ and of $B$ that are of finite index. It follows from this and the c.s. of $A$ (and $B$ ) that any collection of pairwise disjoint sets, each of which is either a conjugacy class or is finite, can be separated by some projection $A \rightarrow A / M$ (or some $B \rightarrow B / N$ ) with $M \in \mathscr{M}$ (or $N \in \mathscr{N}$ ).

Suppose that $x$ is of minimal length in $\{x\}^{P}$, and write $x$ as a cyclically reduced alternating product:

$$
x=u_{1} u_{2} \ldots u_{k} .
$$

The set $S=\left\{u_{1}, \ldots, u_{k}\right\} \cup H$ is finite; and so there is an $(M, N) \in \mathscr{K} \times \mathscr{N}$ such that $A \rightarrow A / M$ separates the elements of $S \cap A$ and $B \rightarrow B / N$ separates the elements of $S \cap B$. Therefore

$$
\pi_{M, N}(x)=\pi_{M, N}\left(u_{1}\right) \pi_{M, N}\left(u_{2}\right) \ldots \pi_{M, N}\left(u_{k}\right)
$$

is a cyclically reduced alternating product in $\boldsymbol{P}_{\boldsymbol{M}, N}$ whose length is equal to $\|x\|$. If $\|x\| \neq 1, \pi_{M, N}(x)$ is therefore of minimal length in its conjugacy class. If $\|x\|=1$, assume for definiteness that $x \in A$. By the minimality of $\|x\|$ in $\{x\}^{P}, H$ and $\{x\}^{A}$ are disjoint; and so $A \rightarrow A / M$ separates $\{x\}^{A}$ and $H$ for some $M \in \mathscr{M}$. In this case, for any $N \in \mathscr{N}$, Theorem 2 shows that $\pi_{M, N}(x)$ is of minimal length in its conjugacy class. 
Let $x$ and $y$ be nonconjugate elements of $P$, each of minimal length in its conjugacy class. By the argument above, there exists $(M, N) \in \mathscr{M} \times \mathscr{N}$ such that $\pi_{M, N}(x)$ and $\pi_{M, N}(y)$ are of minimal length in their conjugacy classes and

$$
\|x\|=\left\|\pi_{M, N}(x)\right\|, \quad\|y\|=\left\|\pi_{M, N}(y)\right\| .
$$

Thus $\pi_{M, N}$ separates $\{x\}^{P}$ and $\{y\}^{P}$ if $\|x\| \neq\|y\|$. Assume now that $\|x\|=\|y\|$; we distinguish three cases.

Case 1. $\|x\|=\|y\|=0$. Let $C_{1}, \ldots, C_{r}$ be the distinct conjugacy classes in $A$ of the form $\{h\}^{A}$ with $h \in H$, and let $D_{1}, \ldots, D_{s}$ be the distinct conjugacy classes $\{h\}^{B}(h \in H)$. Then $\left\{C_{i}\right\},\left\{D_{j}\right\}$ are each pairwise disjoint finite families, and we may choose $(M, N) \in \mathscr{M} \times \mathscr{N}$ such that $A \rightarrow A / M$ separates the $C_{i}$ and $B \rightarrow B / N$ separates the $D_{j}$. If $\pi_{M, N}(x) \sim_{P_{M, N}} \pi_{M, N}(y)$, by Theorem 2 there exist $h_{1}, \ldots, h_{r} \in H$ such that

$$
\pi_{M, N}(x) \sim_{A / M} \pi_{M, N}\left(h_{1}\right) \sim_{B / N} \pi_{M, N}\left(h_{2}\right) \sim \ldots \sim \pi_{M, N}(y) .
$$

By our choice of $(M, N)$,

$$
x \sim_{A} h_{1} \sim_{B} h_{2} \sim{ }_{A} \cdots \sim y,
$$

contradicting $x \sim_{P} y$.

Case 2. $\|x\|=\|y\|=1$. Assume, for definiteness, that $x \in A$, If $y \in A$ choose $(M, N) \in \mathscr{M} \times \mathscr{N}$ so that $\{x\}^{A},\{y\}^{A}, H$ are separated by $A \rightarrow A / M$, and if $y \in B$ so that $\{x\}^{A}, H$ are separated by $A \rightarrow A / M$ and $\{y\}^{B}, H$ are separated by $B \rightarrow B / N$. Then by Theorem 2, $\pi_{M, N}$ separates $\{x\}^{P}$ and $\{y\}^{P}$.

Case 3. $\|x\|=\|y\| \geqslant 2$. Write $x$ as a cyclically reduced alternating product

and put

$$
x=u_{1} \ldots u_{k}
$$

$$
X=\left\{h^{-1} u_{i} \ldots u_{k} u_{1} \ldots u_{i-1} h: h \in H, i=1, \ldots, k\right\} .
$$

Then $X$ is a finite set, and there exists $(M, N) \in \mathscr{M} \times \mathscr{N}$ so that the sets $X$ and $\{y\}$ are separated by $\pi_{M, N}$ and

$$
\left\|\pi_{M, N}(x)\right\|=\|x\|=\|y\|=\left\|\pi_{M, N}(y)\right\| .
$$

Since $\pi_{M, N}(x) \sim_{P_{M, X}} \pi_{M, N}(y)$ implies $\pi_{M, N}(y) \in \pi_{M, N}(X)$ by Theorem 2, it follows that $\pi_{M, N}$ separates $\{x\}^{P}$ and $\{y\}^{P}$.

The proof above used the easy fact that, in a c.s. group, any finite subgroup can be separated from any (disjoint) conjugacy class by a map onto a finite group. It need not be the case that conjugacy classes and arbitrary subgroups are separable, and this provides an obstruction to the c.s. of free products as observed in the lemma below. 
Lemma 5. Suppose $G$ contains a subgroup $H$ and an element $x$ such that $\{x\}^{G}$ and $H$ are disjoint, but are not separated by any homomorphism of $G$ with finite image. Then $(G * G: H)$ is not c.s.

Proof. Write

$$
P=\left(G_{1} * G_{2}: G_{1} \leftarrow H \rightarrow G_{2}\right)
$$

where $G \simeq G_{1}$ under $g \rightarrow g_{1}, G \simeq G_{2}$ under $g \rightarrow g_{2}$, and $H \rightarrow G_{1}, H \rightarrow G_{2}$ are induced by restriction. Then $x_{1}, x_{2}$ lie in different factors of $P$ and are not conjugate to any element of the amalgamated subgroup, whence $x_{1} \sim_{P} x_{2}$. But $\pi\left(x_{1}\right) \sim_{\pi(P)} \pi\left(x_{2}\right)$ whenever $\pi(P)$ is finite, for let $M, N$ be the normal subgroups of $G$ corresponding to $G_{1} \cap \operatorname{Ker} \pi, G_{2} \cap \operatorname{Ker} \pi$ respectively. Put $K=M \cap N$; then $K \triangleleft G,|G / K|<\infty$, and therefore $x K \sim_{G / K} h K$ for some $h \in H$. The homomorphism $\pi$ factors through the map

$$
P \rightarrow P_{1}=\left(G_{1} / K_{1} * G_{2} / K_{2}: G_{1} / K_{1} \rightarrow H K / K \rightarrow G_{2} / K_{2}\right)
$$

induced by the natural projections on the factors, and

$$
x_{1} K_{1} \sim G_{1} / K_{1} h_{1} K_{1}=h_{2} K_{2} \sim_{G_{2} / K_{2}} x_{2} K_{2} .
$$

Consequently $\pi\left(x_{1}\right) \sim_{\pi(P)} \pi\left(x_{2}\right)$.

The same argument shows that $(G * G: H)$ is not residually finite if there exists an $x \in G-H$ such that $\pi(x) \in \pi(H)$ whenever $\pi(G)$ is finite. The HNN extensions constructed by Miller (1971) may be imbedded in amalgamated free products in the usual way, and provide examples of residually finite finitely presented groups with unsolvable conjugacy problem. Thus the class $\mathbf{P}$ of amalgamated free products contains residually finite groups that are not conjugacy separable. Moreover, if each group in $\mathbf{P}$ that has a solvable conjugacy problem were c.s., there would exist a partial algorithm for the conjugacy problem over $\mathbf{P}$; this is impossible. I thank Collins and Miller for settling questions I had raised in an earlier version of this paper.

We turn now to free products with cyclic amalgamated subgroup. We first examine the situation in which the factors are finitely generated nilpotent groups. Blackburn (1965) (or G. Baumslag (1971), p. 6) proves that finitely generated nilpotent groups are c.s. And a theorem of Stebe (1976a) shows that, for any subgroup $H$ of a finitely generated nilpotent groups $G$ and any $x, y \in G$, whenever $\{x\}$ and $H y H$ are disjoint sets, they may be separated by a homomorphism from $G$ onto a finite group. An elegant proof of both these results can be found in Stebe (1976b). Recall that, for a nilpotent group $G$ in which $x$ has infinite order,

$$
x^{r} \sim_{G} x^{s} \text { if and only if } r=s .
$$


LemMA 6. Let $G$ be a finitely generated nilpotent group, and let $H=g p(h), a$ cyclic subgroup of $G$. If $\{x\}^{G}$ and $H$ are disjoint subsets of $G$, then there is a finite quotient group $G / M$ such that the projection $G \rightarrow G / M$ separates $\{x\}^{G}$ and $H$.

Proof. Observe first that Blackburn's Theorem yields the required quotient when $H$ is finite. And if $h$ has finite order $m$ say modulo the center of $G$, then the natural projection $G \rightarrow G / g p\left(h^{m}\right)$ separates $\{x\}^{G}$ and $H$.

We argue by induction on the Hirsch rank, $\mathrm{rk}(G)$, which is the number of infinite cyclic factors in any polycyclic series for $G$. If $\operatorname{rk}(G)=0, G$ is finite and there is no problem. Suppose now that $\operatorname{rk}(G)$ is positive; then there is an element $c$, say, of infinite order and in the center of $G^{\prime}$ (see Baumslag (1971), p. 3). Put $C_{n}=\operatorname{gp}\left(c^{n}\right)$, and note that $\operatorname{rk}\left(G / C_{n}\right)=\operatorname{rk}(G)-1$. By induction and the observation made above, it suffices to separate $\{x\}^{G}$ and $H$ by some projection $G \rightarrow G / C_{n}(n \geqslant 1)$ under the additional assumption that $H \cap \mathrm{gp}(c)=1$.

If $G \rightarrow G / C_{1}$ separates $\{x\}^{G}$ and $H$, we are done; otherwise there exists an element $u \in G$ and integers $i, j$ such that

$$
u^{-1} x u=h^{i} c^{j}
$$

Since $x \sim_{G} h^{i}$, by Blackburn's Theorem there is an epimorphism $\pi: G \rightarrow L$ which separates $\{x\}^{G}$ and $\left\{h^{i}\right\}^{G}$ with $L$ finite. Let $n$ be the order of $\pi(c)$ in $L$. Then $\pi$ factors through the projection $G \rightarrow G / C_{n}$; that is, there is a homomorphism $\sigma: G / C_{n} \rightarrow L$ such that the diagram

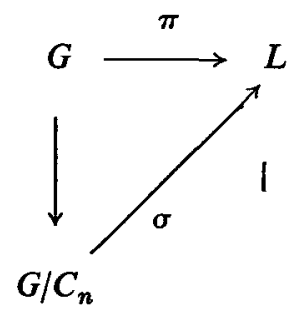

commutes. We claim $G \rightarrow G / C_{n}$ separates $\{x\}^{G}$ and $H$; if not, there is an element $v \in G$ and integers $k, m$ such that

$$
v^{-1} x v=h^{k} c^{n m}
$$

Consequently

$$
h^{i} c^{j} \sim_{G} x \sim_{G} h^{k} c^{n m}
$$

which implies that

$$
h^{i} C_{1} \sim a / C_{1} h^{k} C_{1}
$$


Since $h C_{1}$ has infinite order in the nilpotent group $G / C_{1}, i=k$. Therefore

$$
\pi(x) \sim_{L} \pi\left(h^{k} c^{n m}\right)=\pi\left(h^{k}\right)=\pi\left(h^{i}\right),
$$

contradicting the choice of $\pi$.

THEOREM 7. If $A$ and $B$ are finitely generated nilpotent groups and $H$ is cyclic, then $(A * B: H)$ is c.s.

Proof. We argue by induction on $\operatorname{rk}(A)+\operatorname{rk}(B)$. Observe that, when $H$ is finite, Blackburn's Theorem and Theorem 4 yield the desired conclusion. We may therefore assume that $H$ is infinite; put

$$
H=\mathrm{gp}(h), \quad P=(A * B: H) .
$$

Suppose next that $h^{m}$ is in the center of $P$ for some $m \neq 0$. For each $n \geqslant 1$, set

$$
P_{n}=P / \operatorname{gp}\left(h^{n m}\right) \simeq\left(A / \operatorname{gp}\left(h^{n m}\right) * B / \operatorname{gp}\left(h^{n m}\right): H / \mathrm{gp}\left(h^{n m}\right)\right),
$$

and let $\pi_{n}: P \rightarrow P_{n}$ be the natural projection. The $P_{n}$ are c.s., so that it suffices to separate disjoint conjugacy classes $\{x\}^{P}$ and $\{y\}^{P}$ by some $\pi_{n}$. Observe that for any $x \in P,\|x\|=\left\|\pi_{n}(x)\right\|$ and, if $x$ is of minimal length in $\{x\}^{P}$, so is $\pi_{n}(x)$ in $\left\{\pi_{n}(x)\right\}^{P_{n}}$. Moreover, if $k \geqslant 2$ and $u_{1} \ldots u_{k} \in P$ is cyclically reduced, then its cyclically reduced conjugates are the elements of the finite set

$$
X=\left\{h^{-j} u_{i} \ldots u_{k} u_{1} \ldots u_{i-1} h^{j}: j=1, \ldots, m \text { and } i=1, \ldots, k\right\} .
$$

The argument used to establish Theorem 4 shows that disjoint conjugacy classes $\{x\}^{P}$ and $\{y\}^{P}$ are separated by some $\pi_{n}$ except for the case $x=h^{r}, y=h^{s}$ with $r \neq s$, for which a direct computation shows that $\pi_{|r-s|+1}$ works.

Finally, we may assume that $H$ has infinite order modulo the center of $P$. Since the center of $P$ is the intersection of the centers of $A$ and of $B$, at least one of these factors contains a central element $c$ of infinite order such that $\operatorname{gp}(c) \cap H=1$. For definiteness, assume $c \in A$ and for $n \geqslant 1$ put

$$
P_{n}=\left(A / \mathrm{gp}\left(c^{n}\right) * B: A / \operatorname{gp}\left(c^{n}\right) \leftarrow H \rightarrow B\right) .
$$

Let $\pi_{n}: P \rightarrow P_{n}$ be inducted by the natural projections. Since

$$
\operatorname{rk}\left(A / \mathrm{gp}\left(c^{n}\right)\right)+\operatorname{rk}(B)=\operatorname{rk}(A)+\operatorname{rk}(B)-1,
$$

the $P_{n}$ are c.s. by induction. Observe that any homomorphism $\sigma: A \rightarrow L$ with $L$ finite factors through $A \rightarrow A / \operatorname{gp}\left(c^{n}\right)$ whenever the order of $\sigma(c)$ is a divisor of $n$. Consequently sets separated by maps onto finite quotient groups of $A$ are also separated by the projections $A \rightarrow A / \mathrm{gp}\left(c^{n}\right)$. 
Suppose now that $\{x\}^{P}$ and $\{y\}^{P}$ are disjoint, with $x$ and $y$ of minimal length in their respective conjugacy classes. The observation above and Lemma 6 imply that for some $n, \pi_{n}(x)$ and $\pi_{n}(y)$ are distinct, each of minimal length in its conjugacy class in $P_{n}$, and satisfy

$$
\|x\|=\left\|\pi_{n}(x)\right\|, \quad\|y\|=\left\|\pi_{n}(y)\right\| .
$$

Thus $\pi_{n}$ separates $\{x\}^{P}$ and $\{y\}^{P}$ whenever $\|x\| \neq\|y\|$. We now assume $\|x\|=\|y\|$ and again distinguish three cases.

Case 1. $\|x\|=\|y\|=0$; and so $x=h^{r}, y=h^{s}$ for some pair of distinct integers $r, s$. If $\pi_{1}(x) \sim_{P_{1}} \pi_{1}(y)$, there exist integers $e_{1}, \ldots, e_{m}$ such that

$$
\pi_{1}\left(h^{r}\right) \sim \sim_{\pi_{1}(A)} \pi_{1}\left(h^{e_{1}}\right) \sim \pi_{\pi_{1}(B)} \pi_{1}\left(h^{e_{1}}\right) \sim \ldots \pi_{1}\left(h^{8}\right) .
$$

But $\pi_{1}(h)$ has infinite order in both $\pi_{1}(A)$ and $\pi_{1}(B)$ so $r=e_{1}=e_{2}=\ldots=s$, a contradiction.

Case 2. $\|x\|=\|y\|=1$. Apply Lemma 6 and Blackburn's Theorem to separate $\{x\}^{X},\{y\}^{Y}$, and $H$, where $X=A$ if $x \in A, X=B$ if $x \in B$, and similarly for $Y$.

Case 3. $\|x\|=\|y\| \geqslant 2$. Write

$$
x=u_{1} \ldots u_{k}, \quad y=v_{1} \ldots v_{k},
$$

where both products are cyclically reduced and alternating. For each $i, 0 \leqslant i \leqslant k-1$, denote by $(I: i)$ the set of $k$ equations

$$
(I: i)\left\{\begin{array}{c}
u_{i+1}=h_{0}^{-1} v_{1} h_{1} \\
u_{i+2}=h_{1}^{-1} v_{2} h_{2} \\
\vdots \\
u_{i+k}=h_{k-1}^{-1} v_{k} h_{k}
\end{array}\right.
$$

for elements $h_{0}, \ldots, h_{k} \in H=\mathrm{gp}(h)<P$, with subscripts taken modulo $k$. Denote by $\pi_{n}(I: i)$ the image of the system $(I: i)$ in $P_{n}$. Whenever $u_{i+k}$ and $v_{i}$ are in different factors of $P, \pi_{1}(I: i)$ has no solution in $\pi_{1}(H)$. Whenever $u_{i+k}$ and $v_{k}$ are in the same factor of $P$ but $u_{i+k} \notin H v_{k} H$, Stebe's Theorem guarantees that $\pi_{n}(I: i)$ has no solution in $\pi_{n}(H)$ for some $n$. Consequently there exists a positive integer $n$ such that firstly for each $i=0, \ldots, k-1$,

$(I: i)$ has a solution $h_{0}, \ldots, h_{k} \in H$

if and only if

$\pi_{n}(I: i)$ has a solution $\pi_{n}\left(g_{0}\right), \ldots, \pi_{n}\left(g_{k}\right) \in \pi_{n}(H)$, 
and secondly such that

$$
\pi_{n}\left(u_{1}\right) \ldots \pi_{n}\left(u_{k}\right) \text { and } \pi_{n}\left(v_{1}\right) \ldots \pi_{n}\left(v_{k}\right)
$$

are cyclically reduced alternating products.

We claim that $\pi_{n}(x) \approx P_{n} \pi_{n}(y)$. If not, there exists a cyclic permutation of $\pi_{n}\left(u_{1}\right) \ldots \pi_{n}\left(u_{k}\right)$ which lies in $\left\{\pi_{n}(y)\right\}^{\pi_{n}(H)}$. Relabel so as to assume

$$
\pi_{n}(x) \sim_{\pi_{n}(H)} \pi_{n}(y) .
$$

Consequently $\pi_{n}(I: 0)$ has a solution $\pi_{n}\left(g_{0}\right), \ldots, \pi_{n}\left(g_{k}\right) \in \pi_{n}(H)$ for which also

$$
\pi_{n}\left(g_{0}\right)=\pi_{n}\left(g_{k}\right)
$$

(see the comments following Theorem 2). By our choice of $n$, there exist $h_{0}, \ldots, h_{k} \in H$ satisfying $(I: 0)$. Since $x x_{H} y, h_{0} \neq h_{k}$; and so

$$
\pi_{n}\left(h_{0}\right) \neq \pi_{n}\left(h_{k}\right)
$$

However, $\pi_{n}\left(h_{0}\right), \ldots, \pi_{n}\left(h_{k}\right)$ satisfy $\pi_{n}(I: 0)$ whence

$$
\pi_{n}\left(u_{i}\right)=\pi_{n}\left(g_{i-1}\right)^{-1} \pi_{n}\left(v_{i}\right) \pi_{n}\left(g_{i}\right)=\pi_{n}\left(h_{i-1}\right)^{-1} \pi_{n}\left(v_{i}\right) \pi_{n}\left(h_{i}\right) .
$$

Therefore

$$
\pi_{n}\left(h_{i-1} g_{i-1}^{-1}\right) \sim \pi_{n}\left(v_{i}\right) \pi_{n}\left(h_{i} g_{i}^{-1}\right) .
$$

Since $\pi_{n}(H)$ is infinite cyclic and $\pi_{n}(A), \pi_{n}(B)$ are nilpotent,

$$
\pi_{n}\left(h_{i-1} g_{i-1}^{-1}\right)=\pi_{n}\left(h_{i} g_{i}^{-1}\right)
$$

or

$$
\pi_{n}\left(h_{i}^{-1} h_{i-1}\right)=\pi_{n}\left(g_{i}^{-1} g_{i-1}\right) .
$$

Multiply these equations over $i(1 \leqslant i \leqslant k)$ to obtain

$$
\pi_{n}\left(h_{k}^{-1} h_{0}\right)=\pi_{n}\left(g_{k}^{-1} g_{0}\right)=1,
$$

which is the required contradiction.

The next result of this paper concerns free products with cyclic amalgamation in which the factors are free groups, extending work of Lipschutz (1966) who shows that the conjugacy problem is solvable for such groups, and of Stebe ((1971), Theorem 1) who treats the cyclically reduced elements of length at least two. A result of Baumslag-Higman (Baumslag (1965) Remark, p. 278, or see Lyndon and Schupp (1977), p. 26) asserts that distinct conjugacy classes in free groups are separated by maps onto finite nilpotent groups. Consequently for a free group $F$, the maps $F \rightarrow F / \gamma_{c} F$ separate conjugacy classes, where $\gamma_{c} F$ is the $c$ th term of the lower central series of $\boldsymbol{F}$. 
LEMMA 8. Let $G$ be a free group, and $H$ be a cyclic subgroup of $G$. If $\{x\}^{G}$ and $H$ are disjoint subsets of $G$, there is a positive integer $c$ such that the projection $G \rightarrow G / \gamma_{c} G$ separates $\{x\}^{G}$ and $H$.

Proof. Since $\cap \gamma_{c} G=1$ and $G / \gamma_{c} G$ is torsion-free (Magnus (1935), or see Magnus et al. (1966), chapter 5), there exists an integer $d$ such that $h \notin \gamma_{d} G$ (where $H=\mathrm{gp}(h))$ which implies that $H \cap \gamma_{d} G=1$.

If $\{x\}^{\alpha}$ and $H$ are not separated by the projection $G \rightarrow G / \gamma_{d} G$, there is an integer $n$ such that

$$
x \gamma_{d} G \sim_{G / \gamma_{d} G} h^{n} \gamma_{d} G
$$

Since $x$ and $h^{n}$ are not conjugate in $G$, there is a $c \geqslant d$ such that $G \rightarrow G / \gamma_{c} G$ separates $\{x\}^{G}$ and $\left\{h^{n}\right\}^{G}$ (Baumslag-Higman). If the images of $\{x\}^{G}$ and $H$ in $G / \gamma_{c} G$ are not disjoint, there is an integer $m$ such that

$$
x \gamma_{c} G \sim_{G / \gamma_{c} G} h^{m} \gamma_{c} G .
$$

But $\gamma_{c} G \leqslant \gamma_{d} G$. Consequently $m=n$, contradicting our choice of $c$.

We also require the following consequence of a result of Stebe (1971); a proof is included.

LEMMA 9 (Stebe 1971)). Suppose that $G$ is free, $H$ is a cyclic subgroup, and $x, y \in G$. If $x \notin H y H$, then for some $c>0$ the projection $G \rightarrow G / \gamma_{c} G$ separates $\{x\}$ and $H y H$.

Proof. We may assume that $x \neq 1$ and $y \neq 1$. Put $H=\mathrm{gp}(h)$ and suppose that the images of $\{x\}$ and $H y H$ intersect nontrivially in $G / \gamma_{c} G$ for all $c \geqslant 1$. Thus, for all $c \geqslant 1$, there exist integers $e(c)$ and $f(c)$ such that

$$
x \equiv h^{e(c)} y h^{f(c)}, \text { modulo } \gamma_{c} G .
$$

Choose $d$ so that $\operatorname{gp}(h) \cap \gamma_{d}(G)=1$. When $c \geqslant d$,

$$
\gamma_{c}(G) \leqslant \gamma_{d}(G) \text { and } h^{e(d)} y h^{f(d)} \equiv h^{e(c)} y h^{f(c)},
$$

modulo $\gamma_{d}(G)$, or

$$
h^{e(c)-e(d)} \gamma_{d} G \sim_{G / \gamma_{d} G} h^{f(d)-f(c)} \gamma_{d} G .
$$

Hence the exponents are equal and

$$
e(c)+f(c)=e(d)+f(d) \text { for all } c \geqslant d .
$$


Denote this common value by $m$, and observe that for $c \geqslant d$,

$$
h^{-m} x \equiv h^{-f(c)} y h^{f(c)} \text {, modulo } \gamma_{c} G \text {. }
$$

If $y$ and $h$ commute, $x \in H y H$. Otherwise $f(c)=f(d)$ for $c \geqslant d$, and again, $x \in H y H$.

THEOREM 10. If $A$ and $B$ are free groups and $H$ is cyclic, $(A * B: H)$ is c.s.

Proof. As usual put $P=(A * B: H)$. Free groups and free products of c.s. groups are again c.s. (Stebe (1970)), so that we may assume $A$ and $B$ finitely generated. Choose $d$ so that

$$
\gamma_{d} A \cap H=1=\gamma_{d} B \cap H
$$

and define for $c \geqslant d$,

$$
P_{c}=\left(A / \gamma_{c} A * B / \gamma_{c} B: A / \gamma_{c} A \leftarrow H \rightarrow B / \gamma_{c} B\right)
$$

with epimorphism $P \rightarrow P_{c}$ induced by the natural projections. Recall that, if $x \neq 1$ in the free group $G$, then

$$
x^{r} \sim_{G} x^{s} \text { implies } r=s .
$$

Using the Baumslag-Higman Theorem together with Lemmas 8 and 9, the proof of Theorem 7 applies to show that disjoint conjugacy classes in $P$ are separated by some $P \rightarrow P_{c}$. Since the $P_{c}$ are c.s., so is $P$.

We turn now to HNN extensions and the results communicated by Collins.

THEOREM 11 (Armstrong (1977). If $G$ is free-by-cyclic with nontrivial center, then $G$ is c.s.

Proof. Let $G$ satisfy the short exact sequence

$$
1 \longrightarrow F \longrightarrow G \stackrel{\pi}{\longrightarrow} T \longrightarrow 1,
$$

where $F$ is free and $T$ is cyclic. If $F$ is also cyclic, $G$ is polycyclic and therefore c.s. (see Formanek (1976)); and if $T$ is finite, $G$ is free-by-finite and therefore c.s. (Dyer (1979)). Thus we may assume that $F$ has rank at least 2 and that $T=\mathrm{gp}(t)$ an infinite cyclic group.

Suppose $c$ is a non-trivial element in the center of $G$ and put $C=\mathrm{gp}(c)$. Then $C$ is infinite cyclic ( $G$ is obviously torsion-free) and $C \cap F=1$, whence $G / C$ is freeby-finite and $\pi(c) \neq 1$. 
Suppose that $x$ and $y$ are not conjugate in $G$. If $\pi(x) \neq \pi(y)$, then $\pi$ separates $\{x\}^{G}$ from $\{y\}^{G}$ in the c.s. group $T$. But if $\pi(x)=\pi(y)$, we claim that the projection $G \rightarrow G / C$ separates $\{x\}^{G}$ from $\{y\}^{G}$. Otherwise, $x \sim_{G} y c^{m}$ for some integer $m \neq 0$. Therefore $\pi(x)=\pi(y) \pi(c)^{m}$, whence $\pi(c)^{m}=1$ which contradicts $\pi(c) \neq 1$.

THEOREM 12 (Armstrong (1977)). Let $G$ be a one-relator group with nontrivial center. Then $G$ is c.s.

Proof. Such groups satisfy the hypothesis of Theorem 11 (Baumslag and Taylor (1968), pp. 318-319).

The analog of Theorem 4 for HNN extensions is:

THEOREM 13 (Collins). Let $G$ be c.s. and $H, K$ finite isomorphic subgroups. Then the $H N N$ extension $\left(G, t: t^{-1} H t=K\right)$ is c.s.

ProOf. Let $Q=\left(G, t: t^{-1} H t=K\right)$,

$$
\mathscr{M}=\{M: M \triangleleft G,|G / M|<\infty, H \cap M=K \cap M=1\},
$$

and for each $M \in \mathscr{K}$, let

$$
Q_{M}=\left(G / M, t: t^{-1} H M / M t=K M / M\right) ;
$$

the isomorphism $H M / M \rightarrow K M / M$ is given by the composition

$$
H M / M \approx H / H \cap M \approx H \stackrel{\approx}{\longrightarrow} K \approx K / K \cap M \approx K M / M .
$$

By Theorem 1, the groups $Q_{M}$ are c.s., so that it suffices to show that the induced homomorphisms $Q \rightarrow Q_{M}$ separate disjoint conjugacy classes. The proof parallels that of Theorem 4, using Collins' Conjugacy Theorem (Theorem 3 above), equations (III) through to (VI), the c.s. of $G$ and the finiteness of $H \cup K$.

As observed by Collins, Theorem 13 implies Theorem 4 using the fact that free products of c.s. groups are again c.s. (Stebe (1970), Theorem 3), as follows: let

$$
P=(A * B: A \geqslant C \stackrel{\approx}{\longleftarrow} H \stackrel{\approx}{\longrightarrow} D \leqslant B) \text { and } Q=\left(A * B, t: t^{-1} C t=D\right) .
$$

Define homomorphisms $\alpha: P \rightarrow Q, \beta: Q \rightarrow P$ by

$$
\alpha(x)=\left\{\begin{array}{ll}
t^{-1} x t & \text { if } x \in A, \\
x & \text { if } x \in B ;
\end{array} \quad \beta(x)= \begin{cases}x & \text { if } x \in A * B, \\
1 & \text { if } x=t .\end{cases}\right.
$$


Then $\beta \alpha(x)=x$ for all $x \in P$; and so $u \sim_{P} v$ implies $\alpha(u) \sim_{Q} \alpha(v)$. Thus $Q$ c.s. implies $P$ c.s. On the other hand, in the notation above, $Q \simeq P * \operatorname{gp}(t)$ so that Theorems 7 and 10 imply that certain HNN extensions are c.s. I have chosen to retain the proof of Theorem 4 via amalgamated free products and their structure in that the proofs of Theorems 7 and 10 follow the same general line. The by now familiar non-Hopfian group (a, $\left.t: t^{-1} a^{2} t=a^{3}\right)$ of Baumslag and Solitar (1962) shows that the direct analogues of Theorems 7 and 10 are false for HNN extensions.

\section{References}

S. M. Armstrong (1977), One-relator groups with non-trivial centre (M.Phil. Thesis, Queen Mary College).

G. Baumslag (1963), 'On the residual finiteness of generalized free products of nilpotent groups', Trans. Amer. Math. Soc. 106, 193-209.

G. Baumslag (1965), 'Residual nilpotence and relations in free groups', J. Algebra 2, 271-282.

G. Baumslag (1971), Lecture notes on nilpotent groups (Amer. Math. Soc., C.B.M.S. Regional Conf. Ser. in Math., No. 2).

G. Baumslag and D. Solitar (1962), 'Some two-generator one-relator non-Hopfian groups', Bull. Amer. Math. Soc. 68, 199-201.

G. Baumslag and T. Taylor (1968), 'The centre of groups with one defining relator', Math. Ann. 175, 315-319.

N. Blackburn (1965), 'Conjugacy in nilpotent groups', Proc. Amer. Math. Soc. 16, 143-148.

D. J. Collins (1969), 'Recursively enumerable degrees and the conjugacy problem', Acta Math. 122, 115-160.

J. L. Dyer (1979), 'Separating conjugates in free-by-finite groups', J. London Math. Soc. (to appear).

E. Formanek (1976), 'Conjugate separability in polycyclic groups', J. Algebra 42, 1-10.

A. Karrass, A. Pietrowski and D. Solitar (1972), 'Finite and infinite cyclic extensions of free groups', J. Austral. Math. Soc. 16, 458-466.

S. Lipschutz (1966), 'Generalizations of Dehn's result on the conjugacy problem', Proc. Amer. Math. Soc. 17, 759-762.

R. C. Lyndon and P. E. Schupp (1977), Combinatorial group theory, Ergebnisse der Mathematik Bd. 89 (Springer-Verlag, Berlin-Heidelberg-New York).

W. Magnus (1935), 'Beziehungen zwischen Gruppen und Idealen in einem speziellen Ring', Math. Ann. 111, 259-280.

W. Magnus, A. Karrass and D. Solitar (1966), Combinatorial group theory, Pure and Applied Math. Vol. XIII (Wiley-Interscience, New York-London-Sydney.)

C. F. Miller III (1971), On group-theoretic decision problems and their classification, Ann. of Math. Studies 68 (Princeton University Press, Princeton).

B. H. Neumann (1954), 'An essay on free products of groups with amalgamations', Philos. Trans. Roy. Soc. London Ser. A 246, 503-554.

P. F. Stebe (1968), 'Residual finiteness of a class of knot groups', Comm. Pure Appl. Math. 21, 563-583.

P. F. Stebe (1970), 'A residual property of certain groups', Proc. Amer. Math. Soc. 26, 37-42.

P. F. Stebe (1971), 'Conjugacy separability of certain free products with amalgamation', Trans. Amer. Math. Soc. 156, 119-129. 
P. F. Stebe (1976a), 'Residual solvability of an equation in nilpotent groups', Proc. Amer. Math. Soc. 54, 57-58.

P. F. Stebe (1976b), 'Nests in nilpotent groups', Houston J. Math. 2, 419-426.

Lehman College and The Graduate Center

C.U.N.Y.

New York

U.S.A. 